\title{
To the question of the paradigm shift of psychiatry
}

\section{Rosman $\mathrm{SV}^{* 1}$, Shpak $\mathrm{LV}^{2}$, Maximova $\mathrm{NE}^{3}$}

${ }^{1}$ Physician of functional diagnostics of SBIH, Regional psychoneurological clinic, Russia, Tver.

${ }^{2}$ Professor, Department of Therapy and Cardiology, Postgraduate Education of the SBEI", Tver state medical University" of the Ministry of health of Russia, Tver.

${ }^{3}$ Head of the Department of psychiatry of the SBEI"Tver state medical University" of the Ministry of health of Russia, Tver.

*Corresponding Author: Rosman SV, Physician of functional diagnostics of SBIH, Regional psychoneurological clinic, Tver, Russian Federation, Russia.

Received date: December 20, 2020; Accepted date: January 15, 2021; Published date: January 20,2021

Citation: Rosman SV, Shpak LV, Maximova NE. (2021) To the question of the paradigm shift of psychiatry. International Journal of Clinical Case Reports and Reviews. 6(3); DOI:10.31579/2690-4861/103

Copyright: @ 2021 Rosman SV, This is an open-access article distributed under the terms of the Creative Commons Attribution License, which permits unrestricted use, distribution, and reproduction in any medium, provided the original author and source are credited.

\section{Abstract}

The main problem of psychiatry is that, having gained huge empirical experience in observing psychopathology, it has not become a full-fledged science. Perhaps this happened because Western medicine followed a path different from Eastern medicine - the path of deep study of private pro-phenomena of diseases and the search for their natural causes. However, huge problems lie along this path - the multifactorial impact of the external environment and the limited knowledge of man. As a result, a paradoxical state of affairs was created in psychiatrics and psychology: not a single objective method of research was created that allows you to diagnose mental illness due to the fact that there is still no one's idea of how the brain performs its main function - to think. That is, the reasons for psychiatry's failures lie in the deep crisis of neurophysiology.

Key words: new paradigm of psychiatry; diagnosis of mental illness; new EEG method DAFCAR

Abbreviations: DAFCAR -dispersion of amplitude-frequency characteristics of alpha-rhythm; NGNB - brain neural-glial network

\section{Introduction}

The main problem of psychiatry is that, having gained huge empirical experience in observing psychopathology, it has not become a fullfledged science. Perhaps this happened because Western medicine followed a path different from Eastern medicine - the path of deep study of private pro-phenomena of diseases and the search for their natural causes. However, huge problems lie along this path - the multifactorial impact of the external environment and the limited knowledge of man. As a result, a paradoxical state of affairs was created in psychiatrics and psychology: not a single objective method of research was created that allows you to diagnose mental illness due to the fact that there is still no one's idea of how the brain performs its main function - to think. That is, the reasons for psychiatry's failures lie in the deep crisis of neurophysiology.

Solving all these problems is incredibly difficult, due to established scientific traditions, but it is absolutely necessary objectively - all sensible scientists understand this [16].

Since the ideas that it was the brain that has prevailed is the main creator of all our thoughts and behaviors, researchers have been convinced that the brain is analyzing and synthesizing incoming information - what else? These ideas were the basis of the work of artificial intelligence (AI). However, it is somehow overlooked what technological effort it cost. What complex devices you have to create for this. And most importantly, the resources that have to be used to do so. These are huge energy costs, giant data stores - all to get closer to the intellectual capabilities of the human brain. Unwittingly, the idea that the human brain, having received information, immediately, bypassing the stages of analysis and synthesis, "knows" what to do. Through this idea - the idea that the human brain, as a matter of fact, does not think about anything and cannot cross modern neurophysiologists, although this is what great Russian scientists I.M.Sechenov and I.P.Pavlov - spoke about in their teaching about the reflex activity of the human brain[1,2]. They simply did not have sufficient knowledge of neurophysiology and cybernetics and were unable to complete their ideas. In general, from all this teaching remained only ideas about reflex arc (Fig. 1) 


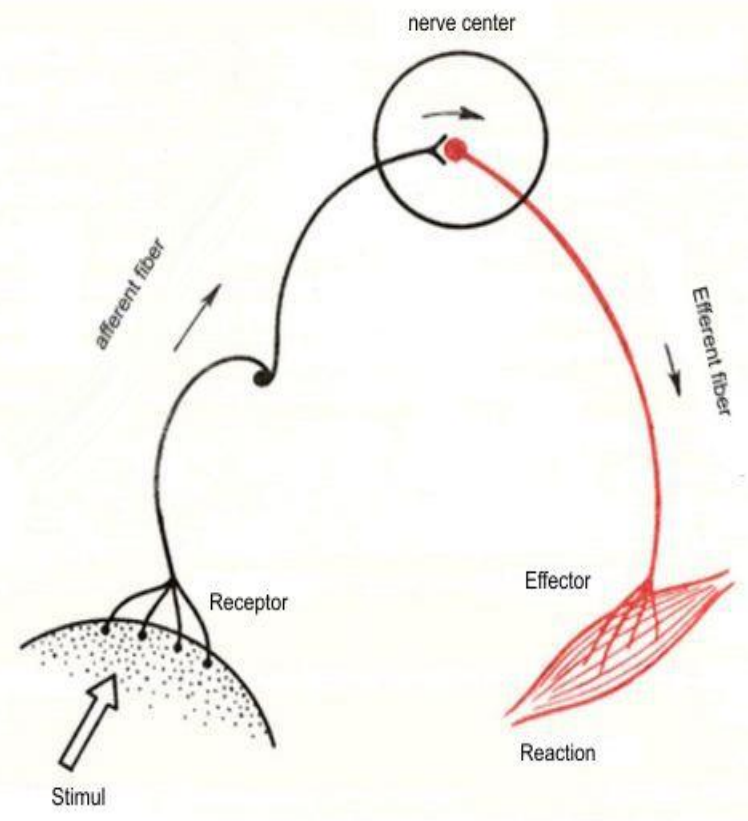

Figure 1: Reflex arch

In many ways, the reflex hypothesis was clarified by P.K.Anokhin[3]. Unlike classical representations of modern scientists, he realized that in nerve centers there is not a simple switching of momentum to executive organs, but a process of "machine coding" of information, which he called afferent synthesis. Based on the results of this synthesis, neural ensembles are created, which are the semantic code of the incoming (afferent) information. Everyone still refers with delight to these advanced, by those times (1930s) ideas, "forgetting" to explain how all this happens. P.K.Anokhin himself was understandably unable to explain this and left the solution to these problems to future generations, creating a theory of functional systems based on the vague incredible computational abilities of neural networks (Fig.2).

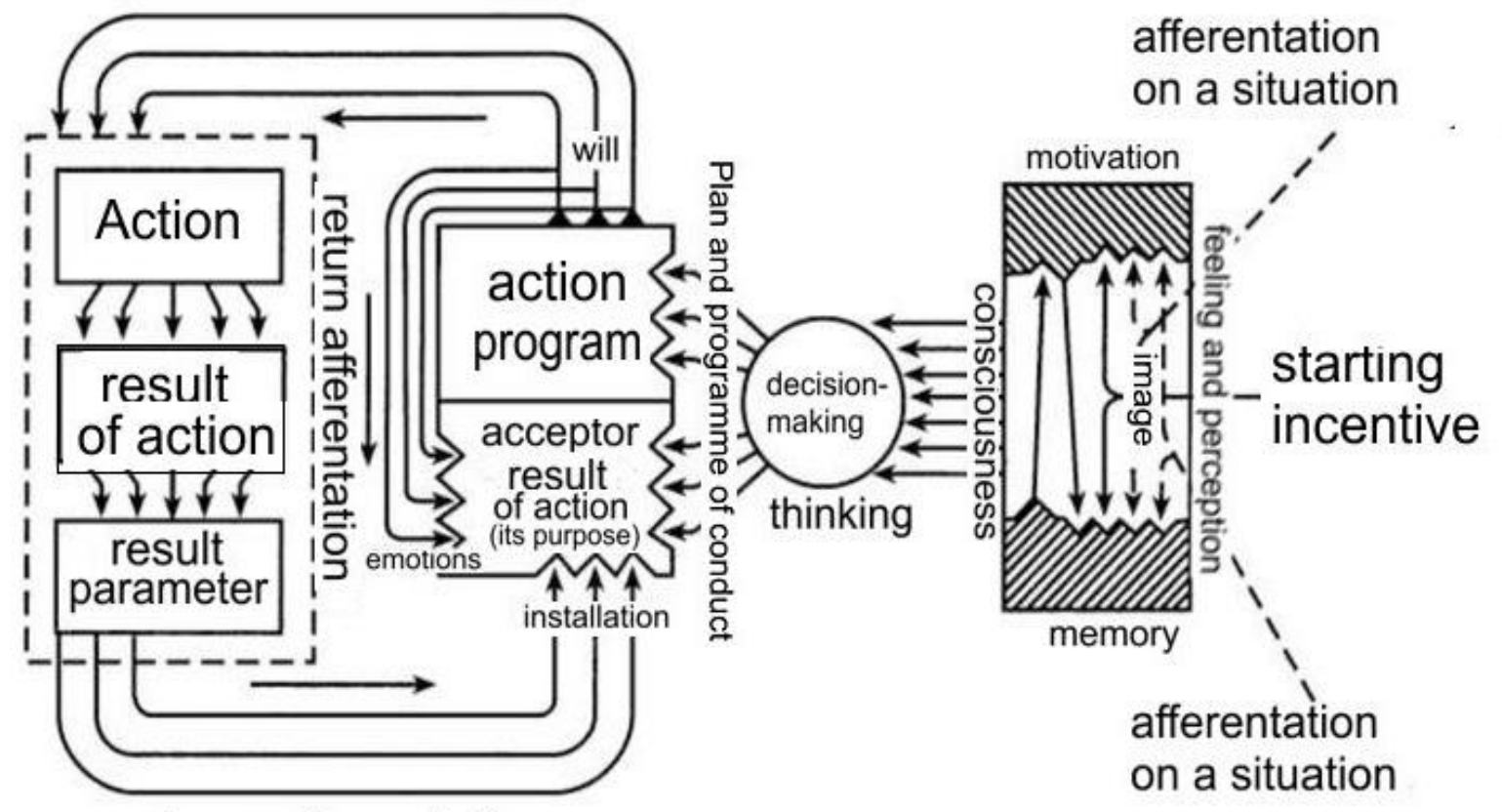

return afferentation 
Theoretical fabrications are good in that they suit everyone, but bad in that it is impossible to build real life on their basis. From the point of view of psychiatry, these remarkable complex constructions do not explain the mechanisms of psychopathology in any way.

It is clearly proven that the process of recognizing afferent information exists - otherwise, how would we distinguish a cat from a dog? So the afferent synthesis process exists. There is also a mechanism for creating neural associations. With the help of neuroimaging devices (fMRI) we can record the occurrence of clusters of excited neurons in the cerebral cortex, which vary depending on the co-maintenance of information. One question remains unclear - how is the process of transforming the results of afferent synthesis (neurophysiological code) into neural associations of a certain configuration (semantic images of information)?

To do this, we need to take a fresh look at the neurophysiological structure of the brain. Without going into functional details, brain tissue is an association of neurons and glial cells, the space between which is filled with a gel-like inter-weft substance - neural-glial brain network (NGBN) (Fig.3).

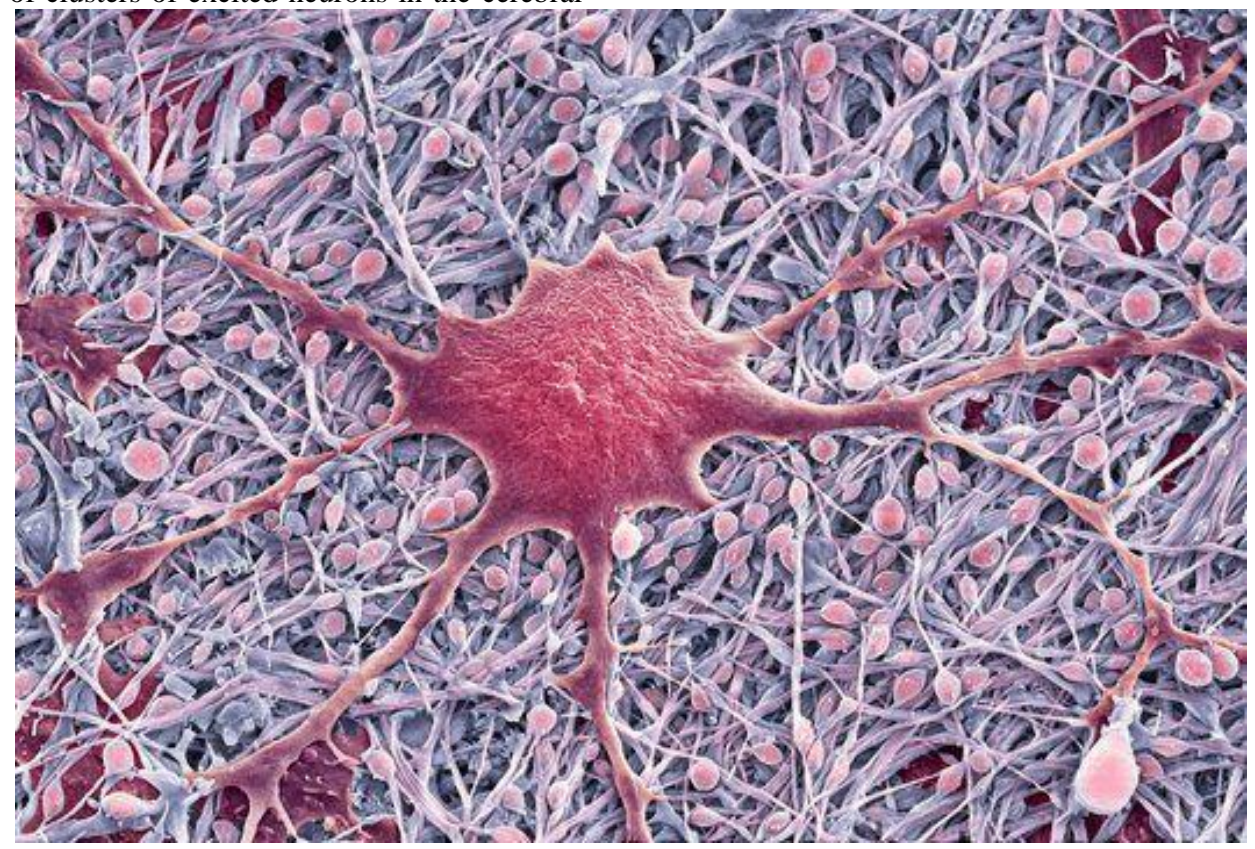

Figure 3: Neural-glial brain network (NGNB). Neuron surrounded by glial cells (photomicrograph).

Until now, glial cells, in the view of neurophysiologists, represent a framework in which neurons performing computational functions are mounted. Trophic (nutritional) conversion and possibility of transformation of pulses, which are supplied to neuron and those transmitted via neural connections, are allowed. What if it's not? According to the old reflex arc concept, the who-beat pulse from the receptor is transmitted directly to the neuron, switching on its synapses, and the neuron processes this pulse and generates an action potential, transferring it by axon to the overlying centers to other neurons. However, if this is the case, all neurons must constantly be in a state of excitation - either from receptors or through inter-neuron connections. Such a system is completely inoperable.

What if neuroglia plays a major role? Excitation from the receptor, entering the NGNB, activates not only the neuron, but also the neuroglia, creating a network-propagating fractal process.

NGNB is an iterative-recursive system. Excitation propagating through this network, moving through its loops, somewhere increasing, and somewhere mutually quenching, creates a complex configuration from neurophysiological conditions called fractal (Fig.4, C).

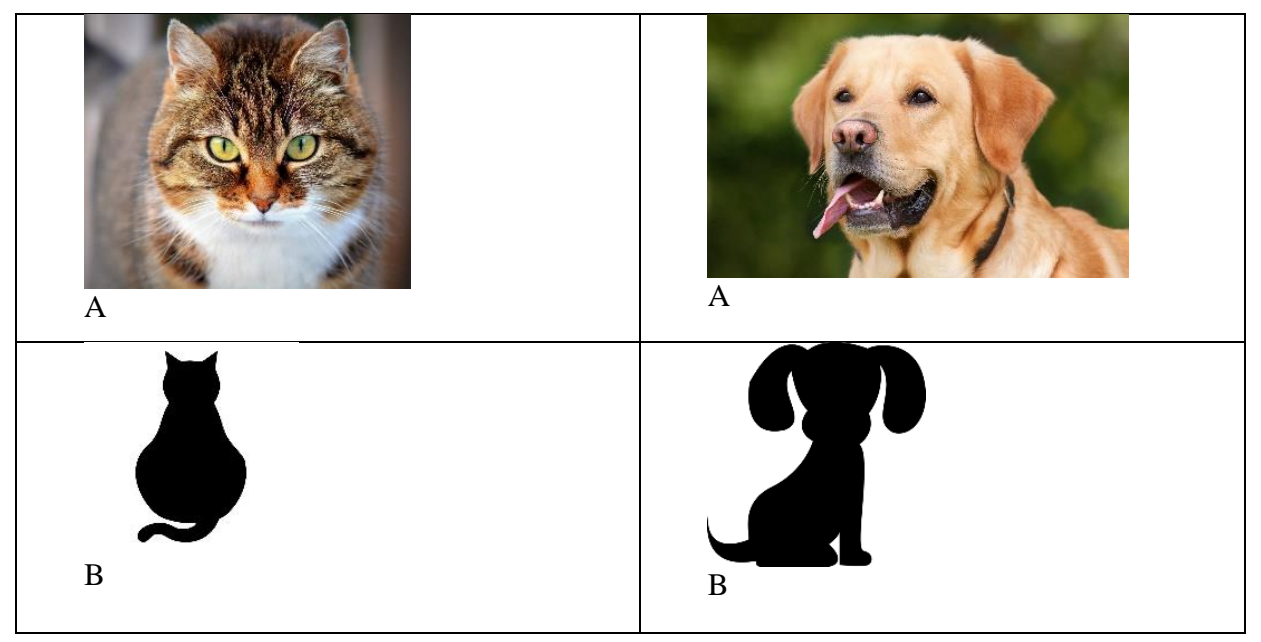




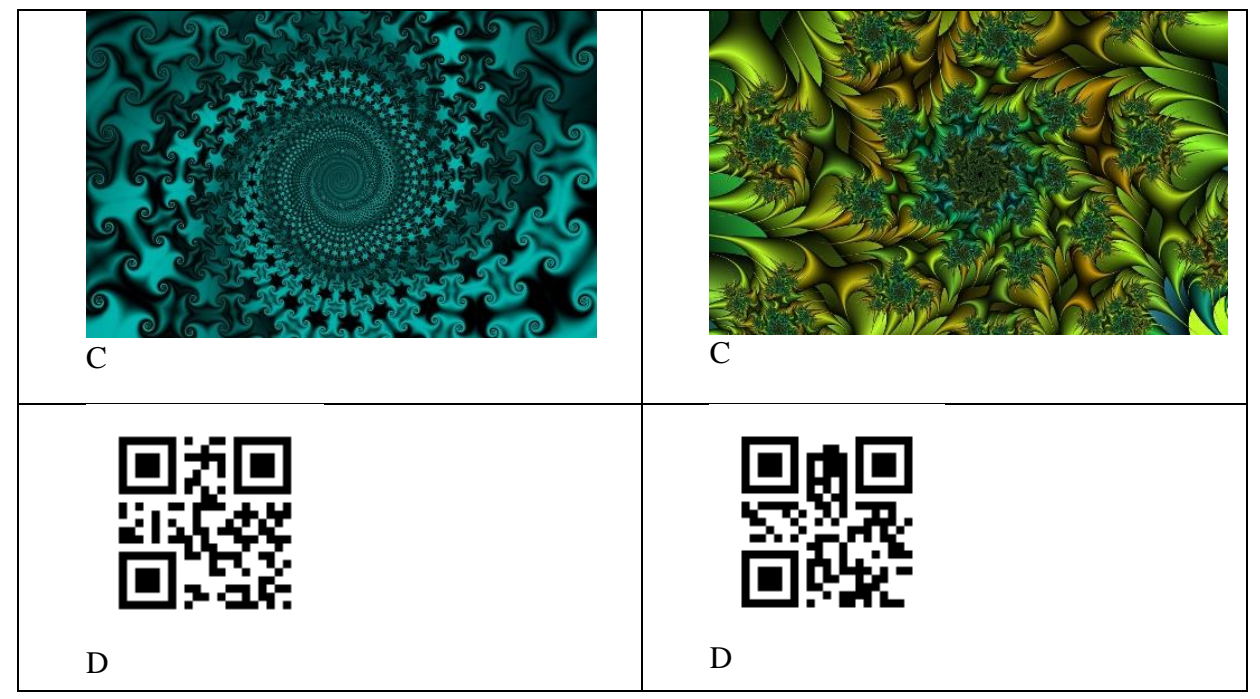

Figure 4: Material object and forms of its semantic representation - A- object, B - conditional image, C - neurophysiological fractal, D - QR-code

A feature of neurophysiological fractal exposure is the fact that various neurophysiological conditions are created around neurons in a certain order located in brain tissue, either favoring action potential generation or preventing it (raising and lowering the action potential threshold. Thus a neural ensemble of neurons generating action potential neurophysiological semantic image (NSI) of the object is created. Conditionally, this can be imagined as the QR-code of the object (Fig.4, D).

However, there is another component of these complex informationinduced neurophysiological changes that are involved in the formation of NSI, which are recorded by EEG - the fluctuating changes in neurophysiological conditions in the interweek matter of the brain that are recorded on the head surface as EEG rhythms. It is completely proven and no one has any doubt that the main rhythm reflecting these changes is the alpha rhythm. So far, the emergence of it and its significance for the process of thinking by official science has never been determined.

Within the present hypothesis, it can be assumed that the alpha rhythm plays the same role as the carrier frequency in the holographic process. As a result, an interference pattern arises from the application of fractal changes to oscillatory changes in the neurophysiological environment. Its purpose is to standardize fractal image and control this process. The fact is that alpha rhythm has strictly standard parameters under normal conditions, but can vary depending on the functional state of the brain or the effect of pathological factors. That's what we're seeing in the case of psychopathology.
Thus, no analysis and synthesis of afferent information occurs in the brain. There is the creation of her semantic image, which launches a set of actions created in the process of previous life, which can be called neurophysiological scenarios (NFS). Rather, it is possible to talk about the mechanism of selection of NFS. How does this mechanism work?

Probably a lot of people know what a Goldberg machine is. Many now compete in creating these increasingly sophisticated devices. The essence of it is that a ball rolls along the inclined yellow, which starts various actuating devices: starts balls, drains water out of the vessel, turns on sound sirens, etc. If we automate the process of returning this machine to its original state, we have before us the simplest model of a reflex arc, from an afferent signal to an efferent response. Now imagine that such machines can be created millions, linked to each other by functional connections and transitions, and we will realize that the functionality of such a system is incredibly high, especially if we realize that the mechanisms of these, the management of them have been refined by evolution for millions of years. The installation of such a Goldberg neurophysiological machine (NMG) takes place during the training of an individual from some initial set of components, which is created in the brain in the form of brain cytoarchitectonics (cell structure), which is genetically standardized within the species. That is why. Despite the fact that each of us has our own personal ideas about each subject, semantic images of them prove to be standardized, and we have the opportunity to understand each other and act in a similar way in similar conditions.

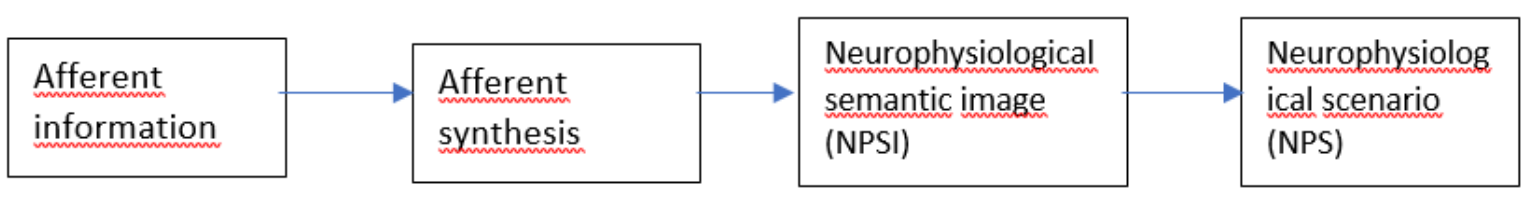

Figure 5: Block diagram of human behavior.

The main requirement for personality behavior is the social acceptability of its NPS.

All these mechanisms work if all links of this flowchart are functionally sound. However, the process of entropy of NGBN interferes here. The essence of it is that NGSMH begins to break down during aging, functional overload or toxic effects, and its functional parameters begin to change and do not meet the necessary criteria. As a result, a distorted NSO is produced, and the NFS ceases to be socially acceptable. This is psychopathology. 
The marker of this process is to change the properties of the alpha rhythm of the EEG. The main parameter of this change is dispersion of amplitudefrequency characteristics of alpha-rhythm of EEG - DAFCAR of EEG[812]. Apparently, there are other, parameters, for example, phasefrequency characteristic of alpha rhythm, but it is not yet developed in relation to psychopathology.

Simplistically, DAFCAR studies the spectral characteristic of the alpha rhythm, which must be normally distributed with the emphasized dominance of one frequency. This means that the alpha rhythm is produced by a strictly defined frequency, and its fluctuations are unacceptable. Normally, the adult alpha rhythm frequency is $9.5-11.5$ Hz. Below this range, mental retardation or dementia (depending on age) is observed. Above - increase of cognitive ability, but above $12 \mathrm{~Hz}$ comes the limit of neurophysiological functionality of neurons, and the person becomes autistic: information is processed at terrible speed, but only excerpt fragments of it are reproduced (classic example - "Rain Man"). Variations in alpha rhythm frequency in the same person are possible, but only in case of acute functional necessity, for example, in case of stress or transition from rest to active action. At rest these oscillations are not more than $\pm 0.25 \mathrm{~Hz}$. An extremely important parameter is the synchronization of DAFCAR values in all parts of the brain, especially in the frontal and occipital lobes. If the difference between the frontal and occipital departments exceeds $0.5 \mathrm{~Hz}$, this results in pronounced deviations in human behavior [7, 13-15].

This question is very important for understanding the essence of schizophrenia. In the human brain there is a function of generation of freedom of will, which also distinguishes man from all other animals. It allows people to do not to the accepted standards, to give out an unusual scenario. This is due to the increase in the amount of human degrees of freedom. It is known that the play of the steering wheel of the car is allowed within 10 degrees, but the completely play-free attachment is not very favorable. This is how a person should have a certain degree of freedom to choose the scenarios of his actions in order to find new optimal solutions to life's problems. Under normal conditions, this capability is realized and functional diagnostics are not recorded. However, when there are deviations in DAFKAR frontal lobes, in light degrees, along with deviations in human behavior, we see higher creativity than others. The degrees of these deviations are varied, and this is expressed in the common observation that all creative personalities are "wrong." Indeed, when we reach a frequency difference between the frontal and occipital lobes of $0.75 \mathrm{~Hz}$ or more, we already notice signs of schizophrenia [10].

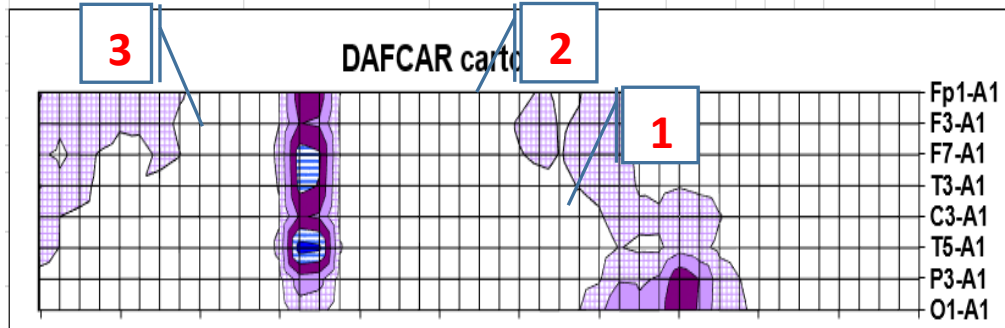

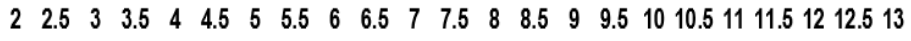

Frequency, $\mathrm{Hz}$, left hemisphere

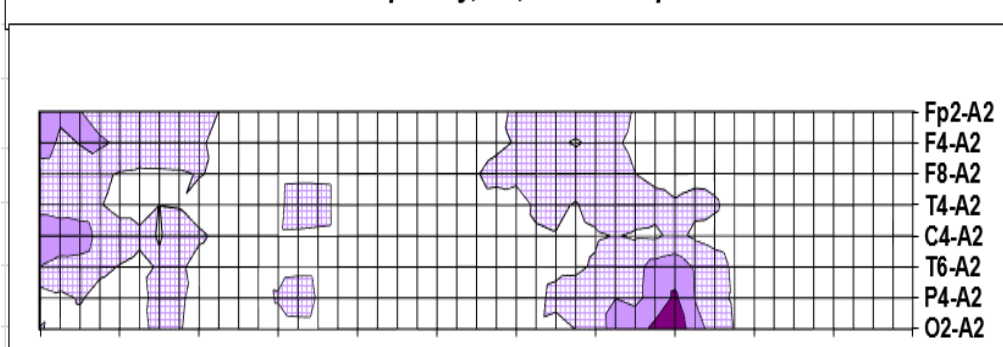

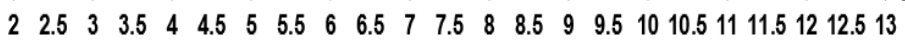
Frequency, $\mathrm{Hz}$, right hemisphere

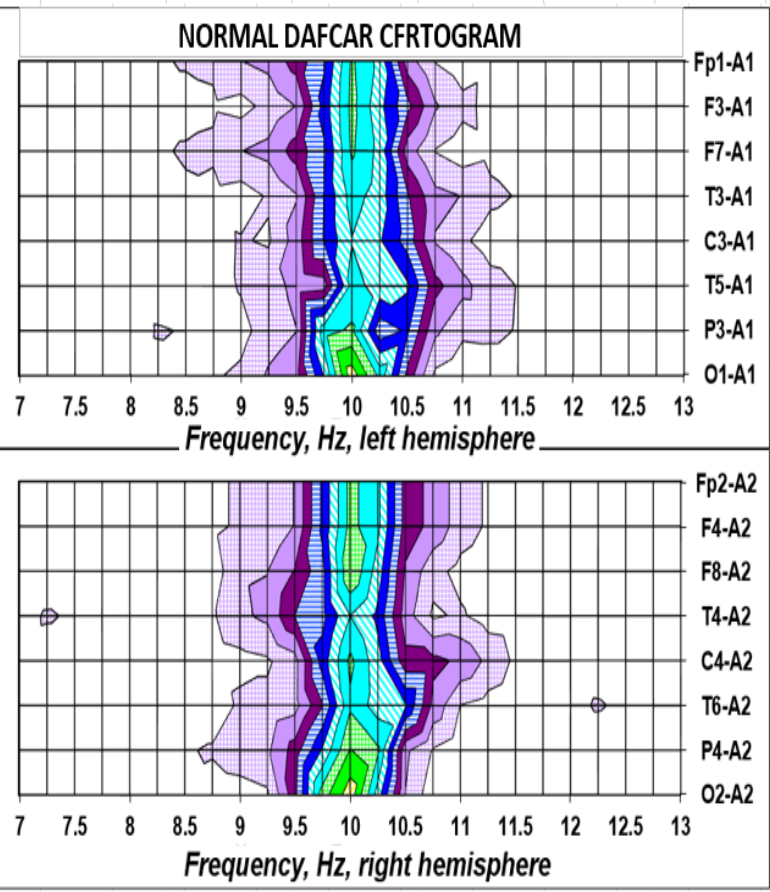

B

Figure 6: Dispersion cartogram for schizophrenia (A) versus norm (B). Pronounced difference of modal frequency in the back of the head (10 Hz) and in frontal sections $(9 \mathrm{~Hz})$ [1-2]; expressed spectrum expansion - 5.5-10 Hz [1-3]

Of course, this case is manifest, but on the basis of the cartogram alone it is not possible to diagnose schizophrenia, although if there are clinical manifestations and obvious deviating behavior, the diagnosis becomes quite obvious.

However, it should be understood that the body has many compensatory possibilities and even with such changes the patient may not be too different from others, although, of course, completely normal behavior is unlikely to differ. His complaints, history, should be examined. Collect information from others and relatives and only then make a judgment.
The issue of schizophrenia and schizophrenia-like symptoms is particularly significant. Schizophrenia is likely an inherited disease; The chances of fully curing it are currently very low, and the level of aggression and autoaggression under it is quite high. At the same time, we often meet with acquired disease of toxic, vascular and traumatic origin, which is very similar to schizophrenia, but with proper targeted treatment of the main cause it is possible to achieve great success. Clinicians do not distinguish these conditions - they can only be distinguished on cartograms. 


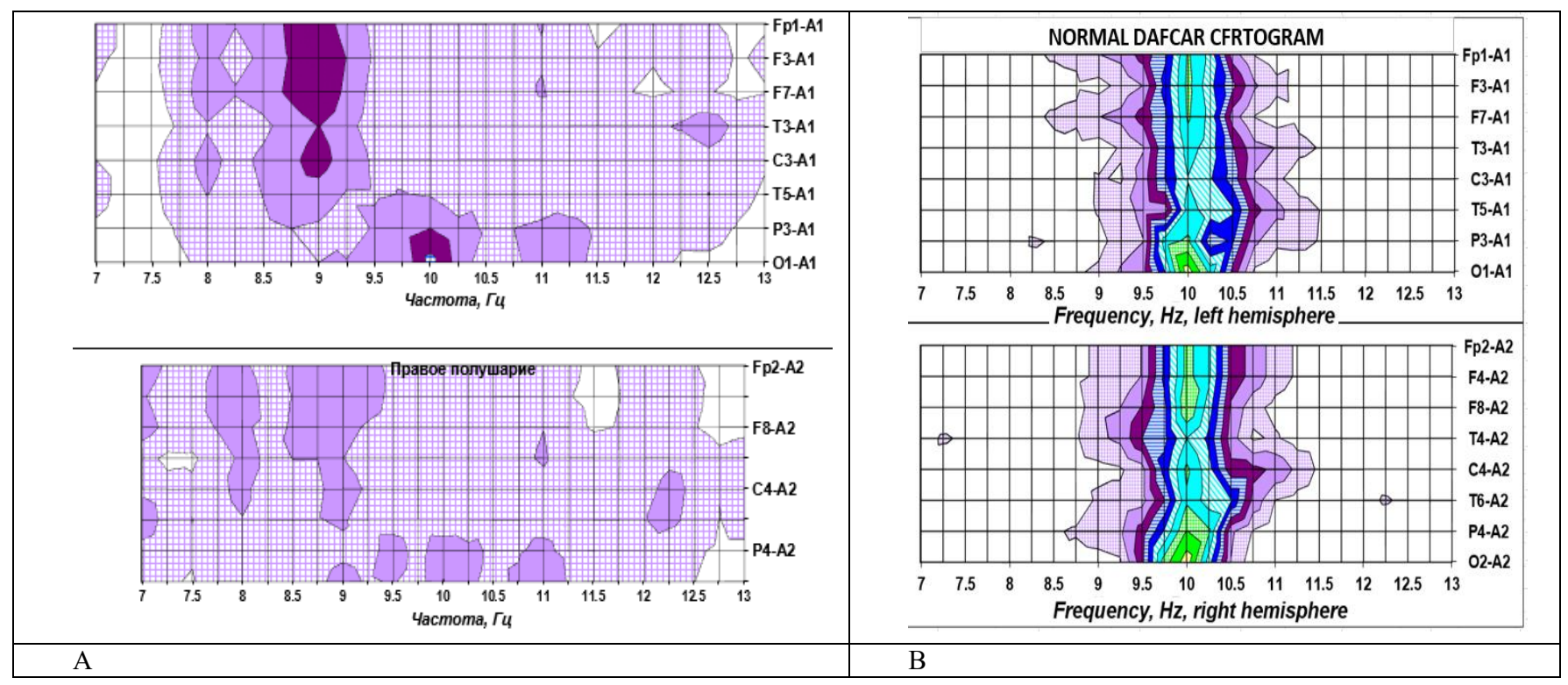

Figure 7: Organic (vascular) disease with schizophrenia-like symptoms (A) in comparison with norm. Difference of modal frequencies in frontal and occipital leads $1 \mathrm{~Hz}$. Rough distinction between hemispheres, as opposed to fig.6

The application of the DAFCAR technique in childhood is particularly valuable, and it is still possible to restore normal neurophysiological changes in the brain. Without going into detail, it can be said that you can only send a child to school if the modal frequency of alpha rhythm in the occipital leads is not lower than $9 \mathrm{~Hz}$. Otherwise, corrective training is needed. If a child has a significant difference in frequencies in the frontal and occipital leads (pituitary), this is a consequence of neurophysiological immaturity of the frontal lobe: usually accompanied by ADHD and other disorders of behavior, enuresis. Such children need special control and correction of neuro-physiological disorders, otherwise this can develop

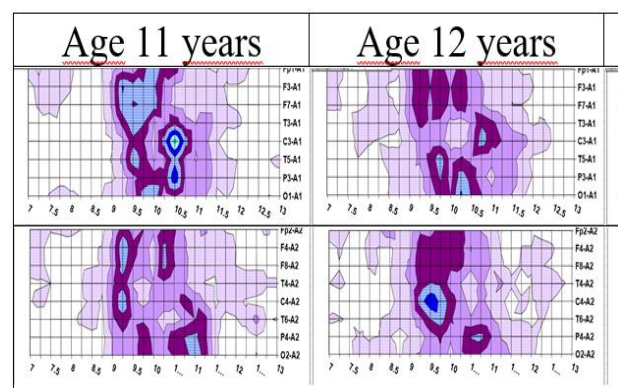

after the puberty period into schizophrenia-like syndrome, or even schizophrenia.

This study is particularly valuable when conducting mass examinations of persons who are subject to increased demands for mental stability. In addition to isolating suspicious patients for hidden pathology, the need for complex psychological testing is significantly reduced. At present, a device has been developed, which in automaton mode calculates DAFCAR parameters and creates cartograms.

Of course, psychiatrists will receive great help by monitoring DAFCAR parameters in dynamics treatment to find optimal therapy.

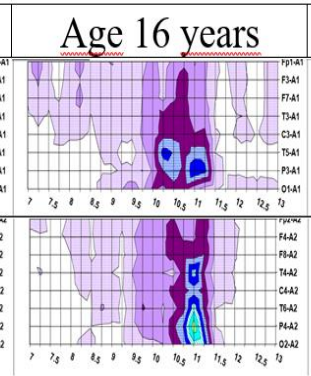

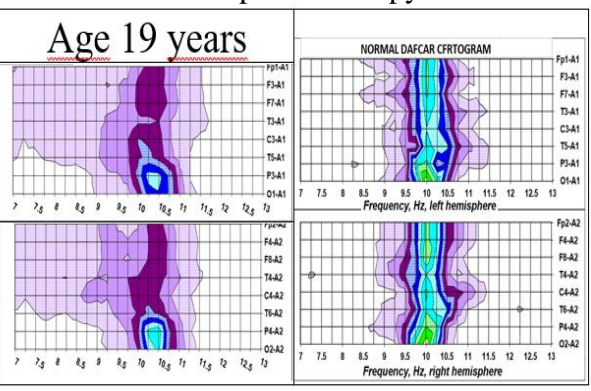

Figure 8: Dynamics of reduction of DAFCAR disorders in adolescents from 11 to 19 years of age with delay of neurophysiological development in comparison with the norm. There is a gradual decrease in the cleavage of the alpha rhythm spectrum. The upper cartogram is the left hemisphere, the lower cartogram is the right hemisphere.

All these empirical observations lead us to a completely logical conclusion: psychopathology should be considered from the perspective of neurophysiological disorders, one of the markers of which is DAFCAR. This does not undo all the empirical experience of psychiatry accumulated over the past 3 centuries. On the contrary, reliance on a natural science basis will give a new impetus in the diagnosis and treatment of mental diseases.

We have already put forward general theoretical prerequisites for systematizing psychopathology in the system "3 + 3" [17].

\section{Informational foundations of human psyche formation:}

1. Inbound Information Afferent Synthesis Process - Information Internalization
2. Creation based on an afferent synthesis of a system of semantic images of the surrounding reality

3. Formation of socially acceptable behaviors based on a system of semantic images in the form of an efferent response

\section{The main criteria for a person's mental state are:}

1. Normal state of the psyche - on the basis of standard afferent synthesis, a system of congruent semantic images of the surrounding reality is created for this type, on the basis of which socially acceptable models of behavior of the individual are created

2. Psychopathology is a violation of the normal process of afferent synthesis, as a result of which pathological polysemantic images 
of the surrounding reality is created, on the basis of which socially unacceptable patterns of behavior of the individual are formed

3. The marker of the process of disruption of afferent synthesis is the degree of entropy of the NGNB, manifested in DAFCAR.
The logical continuation of these theoretical provisions was the neurophysiological systematization of psychopathology, based on the neurophysiological marker - DAFCAR.

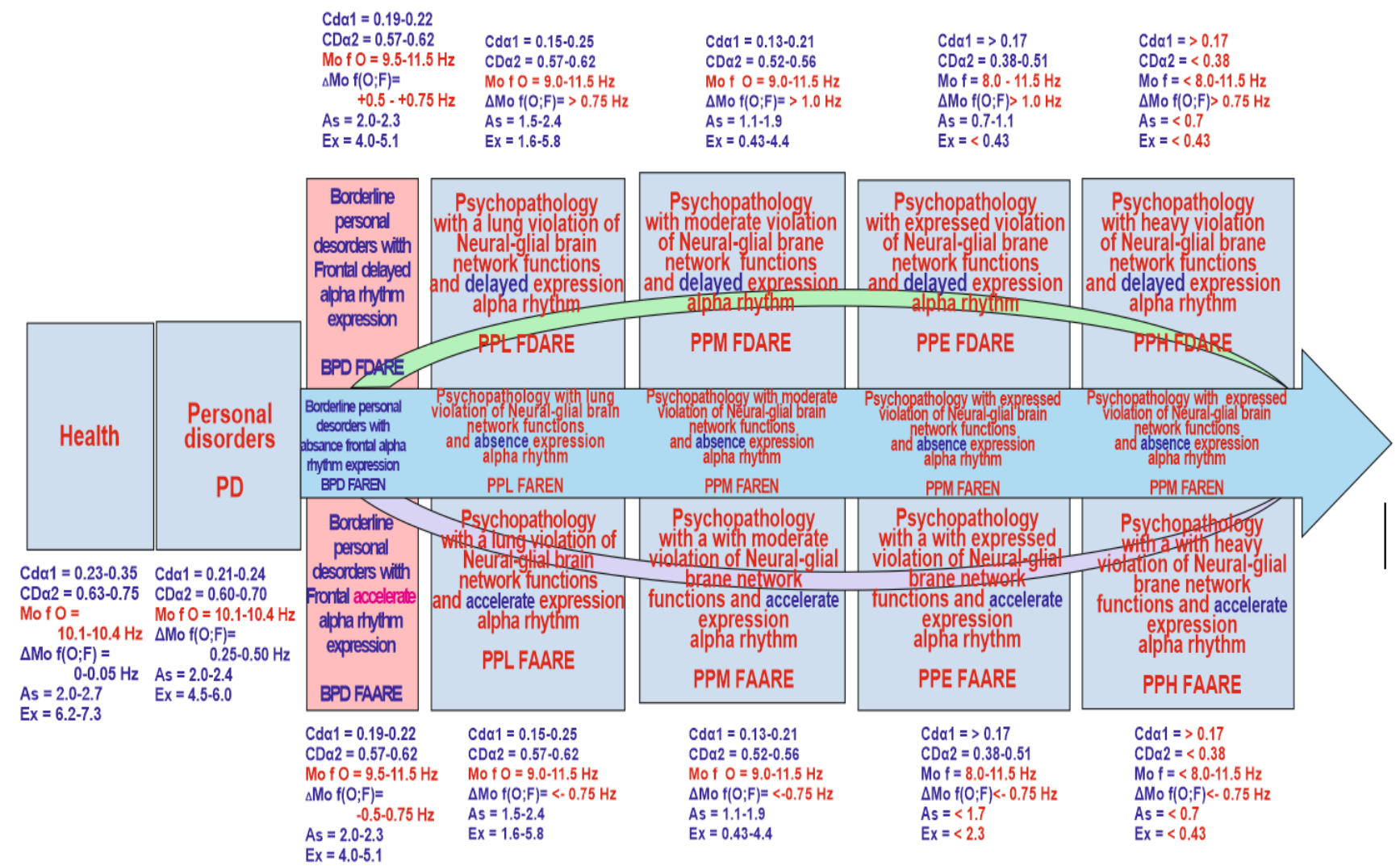

Figure 9: Neurophysiological systematization of psychopathology.

General systematization is based on historically very deep ideas about the human mentality. According to them, a person's mentality can be divided into 4 main groups: perfect (mental health), easily changed (in modern systematization - personality disorders), borderline mental disorders and psychopathology.

The systematization of psychopathology consists in the division of DAFCAR changes with a general deceleration of alpha rhythm in all leads (built along the blue arrow), and regional decelerations or accelerations of alpha rhythm (delayed and accelerated frontal expression), mainly in the frontal departments (changes located above and below the blue arrow. At the same time, the deceleration and acceleration of the alpha rhythm can be both monomodal (without a pronounced spread of indicators around the central value) and dispersive (with a pronounced spread of indicators around the central value). The proposed systematization is presented in Fig. 9.

The values of the coefficients presented on it are set forth in theoretical articles on DAFCAR. The basic principle is very simple: the increase in entropy (disorganization) of the neural network leads to a slowdown in alpha rhythm and a decrease in dispersion coefficients. Most often, pronounced slows in alpha rhythm and pronounced dispersion lead to dementia (mental retardation). Regional alpha rhythm disorders in frontal leads (deceleration and acceleration) most often lead to diseases of the "schizoid circle." Naturally, systematization can be expanded on the basis of old categorical diagnostic criteria. The main thing we can get from the application of new systematization is an understanding of the mechanisms of mental disorders and which disorders should be eliminated in order to bring the patient's consciousness back to normal. Thus, it becomes possible to treat not a diagnostic label, but the basis of the disease and the patient himself.

\section{Conclusions}

The goal of changing the paradigm of psychiatry and psychological sciences is:

1. Changing fundamental ideas about the mechanism of the information process in the brain and affirming the decisive role of neurophysiological processes in the human body in the formation of the human psyche and personality

2. Establishing a direct genetic link between the eastern model of medicine affirming the psychosomatic and informational unity of the human body and the categorical western model creating the semantic image of the disease in the form of diagnosis from a collection of symptoms

3. Obtaining objective data explaining the genesis of human behavioral features

4. Early diagnosis of the level of neurophysiological maturation of the brain in children with the aim of early correction of 
deviations in this process and development of optimal tactics of life strategy of patients

5. Monitoring in the process of developing new "physiological" methods for correcting disorders in NGSGM

6. Early diagnosis of probability of deviant behavior in a population for the purpose of early prevention of unlawful violent acts

7. Screening among the contingent of applicants for work in conditions of high public risk (authorities, pilots, dispatch service, drivers of public vehicles, military personnel, law enforcement officials)

8. Deep penetration into the essence of the mentality of criminals in order to prevent illegal actions and mass terrorist acts

9. Early diagnosis of mental illness and behavioral disorders; dynamic monitoring of pathological process and therapy; objective control over development of new methods of treatment of psychopathological disorders.

\section{References}

1. Anokhin P.K. (1998) Selected works. Cybernetics of functional systems/under the general ed. Prof. K.V. Sudakova (comp. V.A. Makarov). - M.: Medicine.

2. Ivanov LB. (2016) Psyco-phisiological interpretation of the functional state of the brain using EEG. Bullet. Of clin. Neurophysiology. (1): 5-26.

3. Iznak A. F. (1989) Modulation of sensory-motor human activity on the background of alpha-rhythm EEG // Problems of development of scientific research in the field of mental health. p. 3-24.

4. Kustubayeva A.M. (2012) Age dynamics of rhythms of electric activity of a brain. Level uneasiness and EEG indexes. Expert psychology. (3): 5-20.

5. Pavlov I.P. (1952) Physiology of the nervous system//I.P.Pavlov, I.M.Sechenov, N.E.Vvedensky. L., V.4.

6. Rosman SV, Maximova NE. (2018) Depression in the Context of Search of a New Paradigm in Psychiatry. Clin Depress.
7. Rosman S, Kurakhina O. (2017) Violent Crime in the Context of Entropy Neuron-Glial Networks of the Brain. Glob J Add \& Rehab Med. 2(5): 555599.

8. Rosman S. (2018) The «System $3+3$ » in a Problem of Searching of a New Paradigm in Psychiatry. Psychol Psychother Res Stud .1(5). PPRS.000522.

9. Rosman S. (2017) The Theoretical Foundations of Dispersion of Amplitude-Frequency Characteristics of the Alpha Rhythm of the EEG. Glob J Add \& Rehab Med.; 2(3): 555587.

10. Rosman S.V. (2013) Diagnostic capabilities of dispersion mapping the alpha rhythm of the electroencephalogram. Mental health. 6:64-69.

11. Rosman S.V. (2014) Integral indexes of dispersion of amplitude-frequency characteristics of an alpha rhythm of an EEG in diagnosis of mental diseases. Mental health. (1): 132135.

12. Rosman S.V., Shpak L.V. (2013) New approaches to the assessment of polymorphism of the alpha rhythm EEG in psychiatric disorders. Ment.Health. 2:39-44.

13. Rosman S.V., Shpak L.V. (2013) New approaches to the assessment of polymorphism of the alpha rhythm of the electroencephalogram with mental illness. Mental health. 2 3944.

14. Rosman SV. (2017) Borderline Personality Disorder in the Context of Entropy Neuron-Glial Networks of the Brain. Glob J Add \& Rehab Med.; 2(4): 555595.

15. Rosman SV. (2017) Burnout Syndrome in the Context of Entropy Neuron-Glial Networks of the Brain. Glob J Add \& Rehab Med. 3(3): 555611.

16. Rosman SV. (2017) Pedophilia in the Context of Entropy Neuron Glial Networks of the Brain. Glob J Add \& Rehab Med.; 4(2): 555632.

17. Sanislow C.A. (2016) Updating the research domain criteria. World Psychiatry. 15 (3): 222-223.

18. Sechenov I.M. Selected works. M.; L.., 1952. 